\title{
PCR identification and phylogenetic analysis of the medically important dust mite Suidasia medanensis (Acari: Suidasiidae) in Malaysia
}

\author{
F. C. L. Ernieenor ${ }^{1} \cdot$ G. Ernna ${ }^{1}$ - A. S. Jafson ${ }^{1} \cdot$ A. Mariana ${ }^{1}$
}

Received: 25 January 2018 / Accepted: 17 August 2018 / Published online: 27 August 2018

(c) The Author(s) 2018

\begin{abstract}
The occurrence of Suidasia medanensis (=S. pontifica) mites in Malaysian house dust was first reported in 1984. The taxonomy of this storage mite is, however, quite confusing. Therefore, we need an accurate identification to resolve morphological problems due to its minute size and some overlapping characters between species. The purpose of this study was to demonstrate the application of partial mitochondrial cytochrome c oxidase subunit I (COI) sequences for the identification of $S$. medanensis by PCR. Identity of the mite was first determined by observing morphological characters under a light microscope. Genomic DNA of $S$. medanensis mites was successfully extracted prior to PCR and DNA sequencing using COI universal primers. The length of the COI sequences obtained was $378 \mathrm{bp}$. BLAST analysis of amplicon sequences showed that local S. medanensis COI region had 99\% maximum identity with S. medanensis nucleotide sequence (AY525568) available in the GenBank. As the phylogenetic tree generated indicated, $C O I$ sequences from this study were clustered with $S$. medanensis from Korea and the UK in one major clade, supported with high bootstrap value $(>85 \%)$. Results of the phylogenetic analysis of this $C O I$ gene were congruent with the morphological identification and provided strong support for a single clade of local $S$. medanensis.
\end{abstract}

Keywords Suidasia medanensis $\cdot \mathrm{COI} \cdot$ Phylogeny $\cdot$ Neighbor-joining $\cdot$ Astigmata

\section{Introduction}

Families of mites that have been identified in house dust are Pyroglyphidae, Acaridae, Glycyphagidae, Chortoglyphidae, Suidasiidae and Echimyopodidae (Puerta et al. 2005; Cui 2014). Amongst these, Suidasiidae is one of the most important families due to its economic and public health impact. Of this family, the genus Suidasia comprises 18 species (Krantz et al. 2009), which are commonly found in house dust and stored food products in tropical countries such as India, Taiwan and Thailand (Chmielewski 2009). Suidasia

F. C. L. Ernieenor

erniee@imr.gov.my

1 Acarology Unit, Infectious Diseases Research Centre, Institute for Medical Research, Jalan Pahang, 50588 Kuala Lumpur, Malaysia 
medanensis (=S. pontifica) Oudemans (Acari: Astigmata: Suidasiidae) was reported for the first time in Malaysia in house dust samples in 1984 (Ho and Nadchatram 1984). The mites were widely distributed as such and they were reported to infest many stored products including grains, cheese, nuts, milk powder and rice grain and flour (Ho and Nadchatram 1985; Ho and Mariana 1994; Ho 1996; Mariana et al. 2000, 2010).

The species involved is of medical significance as it has the ability to produce potent allergens causing allergic reactions in humans (Chmielewski 2009; Mariana et al. 2011). Whole-body extract of $S$. medanensis has at least 14 allergens, suggesting an important allergenic load and sensitizing potential (Puerta et al. 2005). To determine the clinical relevance of $S$. medanensis, some studies have shown a high percentage of asthmatics patients to be sensitized to the mite species (Mariana et al. 2002; Yap et al. 2014). Furthermore, ingestion of food prepared from flour contaminated with these mites was reported to cause anaphylaxis with symptoms of breathlessness and facial angiodema to the allergic patients (Sanchez-Borges et al. 1997, 2009).

The quality of mite allergens is depending on the production of body proteins and digestive enzymes that are discharged within fecal pellets (Calderon et al. 2015). Accurate species identification is important to avoid contamination of other foreign mites for standardization of the allergen production (Frati et al. 2012). Dust mites have been identified mainly based on morphological characteristics of the adult stage. However, it is difficult to identify species by their phenotypic traits alone due to their microscopic size ( $\mathrm{Li}$ et al. 2015) and similarities of characters (Beroiz et al. 2014). There is also no figure available for descriptions of morphological polymorphism in adults (Vargas et al. 2005). Moreover, immature mites often lack obvious diagnostic characters ( $\mathrm{Li}$ et al. 2010) for species confirmation and they often must be reared until adult stage for accurate identification. The taxonomy of the genus Suidasia is somewhat confusing and the identification of species was unresolved due to variability of morphological characters that overlap between closely related species (Mercado et al. 2001).

An accurate molecular tool for species identification would be highly valuable. Progress in molecular biology techniques has enabled the identification and the determination by DNA sequence analysis of phylogenetic relationships of a range of mite species. The central part of the mitochondrial cytochrome c oxidase subunit I (COI) region is highly conserved and has often been used for identification of astigmatid mites and for determination of their intra- and interspecific variation. This is due to their variation in the $C O I$ sequences and higher rates of molecular evolution between closely related mite and tick species (Yang et al. 2011; Erster et al. 2013; Lv et al. 2014; Khaing et al. 2014). The purpose of the present study was to demonstrate the application of partial mitochondrial COI sequences for identifying $S$. medanensis by PCR. Genetic profile of this mite was also discussed based on molecular phylogeny using the COI region. Information on the genetics of domestic mites is important to understand the population dynamics of laboratory cultures and in mite control.

\section{Materials and methods}

\section{Culture of mites}

Suidasia medanensis mites were first obtained from house dust sample collected from Pahang, Malaysia. Those colonies were established in the 1980's and maintained in the 
Acarology Unit, Institute for Medical Research, Malaysia. Colonies consisting of mixed stages of mites were maintained at $25 \pm 2{ }^{\circ} \mathrm{C}$ and $75 \pm 2 \%$ relative humidity. Purity of the colonies was checked by microscopical examination of a few randomly mounted specimens. The identity of mites was determined by analyzing external morphological characteristics (Hughes 1976; Fain and Philips 1978) using a phase contrast microscope (Nikon, Japan).

\section{Harvest of mites and DNA extraction}

The mite cultures were harvested with a 'heat escape' method in which mites migrated from the sieve located on top of a funnel to a 1-ml sterile collection Eppendorf tube at its bottom. The collected bodies (ca. $100 \mathrm{mg}$ ) were immediately resuspended in $25 \mu \mathrm{l}$ of phosphate buffered saline (PBS) and $75 \mu \mathrm{l}$ of animal tissue lysis (ATL) buffer. The mites were then crushed using sterile plastic tips for $5 \mathrm{~min}$ prior to adding $20 \mu \mathrm{l}$ of Proteinase K. The following steps were performed using the manufacturer's supplementary protocol for the Qiagen DNeasy ${ }^{\circledR}$ Kit for animal tissues (Qiagen, Germany). Purity and concentration of DNA was determined using Thermo Scientific NanoDrop ${ }^{\mathrm{TM}} 1000$ spectrophotometer (Wilmington, DE, USA). The extracted DNA was either directly used as a template for PCR amplification or stored at $-20{ }^{\circ} \mathrm{C}$ until further usage.

\section{PCR amplification}

Amplification of the $C O I$ gene region was performed using the following universal primers: forward (5'-GTT TTG GGA TAT CTC TCA TAC-3') and reverse (5'-GAG CAA CAA CAT AAT AAG TAT-3') which amplified the central region of COI. PCR was performed in $50 \mu \mathrm{l}$ total volume containing $10 \mu \mathrm{l}$ of DNA, $25 \mu \mathrm{l}$ of $2 \mathrm{X}$ Taq PCR Master Mix (Qiagen), $2.5 \mu \mathrm{l}$ of each primer $(10 \mu \mathrm{M})$, and $10 \mu \mathrm{l}$ nuclease-free water. For each PCR reaction, a negative control containing sterile double distilled water and a positive control containing mite DNA (A. ovatus) were included. The mixture was amplified by the following conditions: initial denaturation at $94{ }^{\circ} \mathrm{C}$ for 3 min followed by 35 cycles denaturation at $94{ }^{\circ} \mathrm{C}$ for $30 \mathrm{~s}$, annealing at $50{ }^{\circ} \mathrm{C}$ for $30 \mathrm{~s}$, elongation at $72{ }^{\circ} \mathrm{C}$ for $1 \mathrm{~min}$ and a final elongation step at $72{ }^{\circ} \mathrm{C}$ for $7 \mathrm{~min}$. PCR was carried out in an Eppendorf Mastercycler Personal machine. Amplicons were analyzed by $1.5 \%$ agarose gel electrophoresis and positive bands were viewed under an ultraviolet trans-illuminator (at $254 \mathrm{~nm}$ wavelength).

\section{DNA sequencing}

The DNA fragment was excised from the gel using a sterile sharp gel cutter and purified using 5 Prime PCR Agarose Gelextract Mini Kit (Hamburg, Germany) according to the manufacturer's protocol. All PCR products were then sent for DNA sequencing to First Base Laboratory, Malaysia. The sequencing was bi-directional for all specimens and the primer combination for this step was the same as that used in the PCR amplification.

\section{Gene sequence analysis}

Multiple sequence alignment was carried out using the Clustal-W program of BioEdit (Hall 2005). Identified sequences were compared with available sequences of dust mite 
previously deposited in GenBank databases using the Basic Local Alignment Search Tool (BLAST) program. In addition, two $S$. medanensis sequences that were available in GenBank (Accession Nos. AY525568 and KJ820779) were aligned simultaneously as analysis background and species control. In this analysis, Tyrophagus putrescentiae (GenBank Accession No. EF527826) was selected as an outgroup for COI gene.

\section{Phylogenetic analysis}

Phylogenetic analyses were performed using Molecular Evolutionary Genetic Analysis (MEGA) software v.4.0 with the neighbor-joining (NJ) method. Bootstrap values were obtained from 1000 replicates. Sequence divergence was calculated using MEGA among taxa, based on Kimura-2-parameter (K2P) distance.

\section{Results}

The morphology of $S$. medanensis can be distinguished from other mite species by the presence of a dorsal transverse groove that divides the propodosoma from the hysterosoma (Fig. 1a). The ventral part of the mite is finely wrinkled or covered with scale-like cuticle (Fig. 1b). Their legs are stout with claw at the end of the tarsus (Fig. 1b). The molecular analysis matched its morphological identification. The primer combination of COI-F and COI-R was demonstrated to amplify mtDNA of the mite. Length of the obtained PCR product of the COI was approximately 378 bp in all examined samples (Fig. 2). The BLAST analysis of amplicon sequences indicated that the COI region of the local $S$.
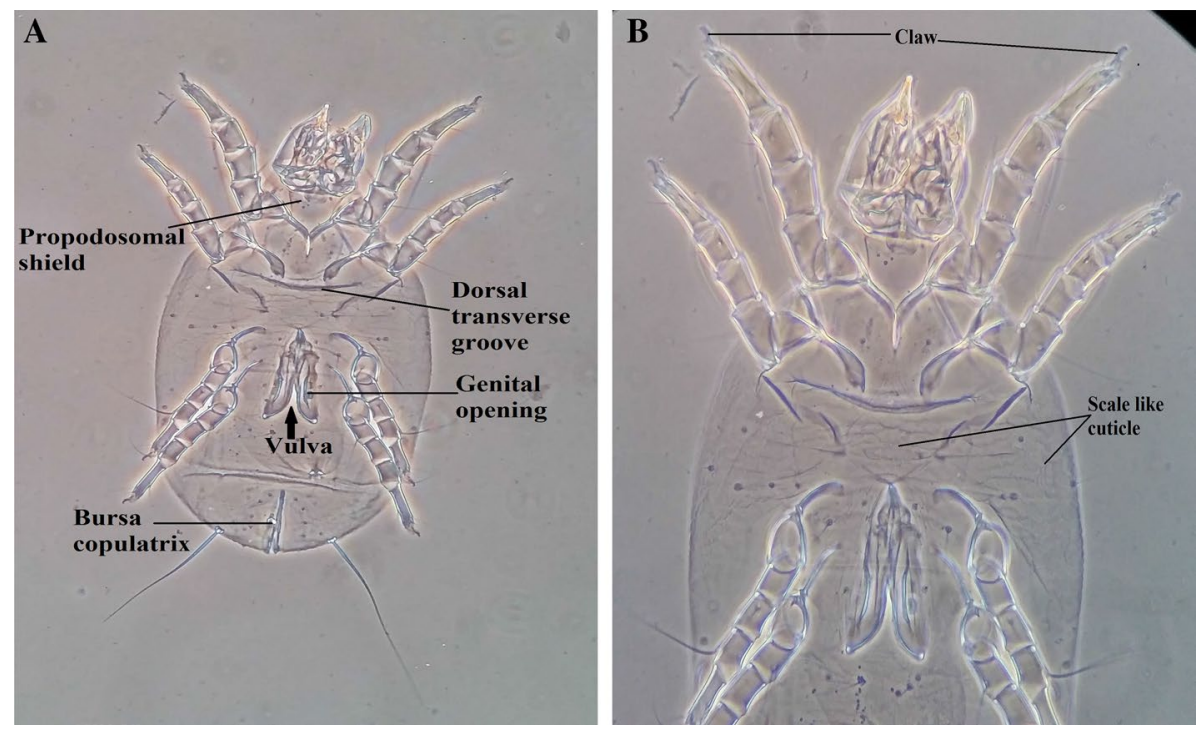

Fig. 1 Morphological characters of Suidasia medanensis female. a Transverse groove and propodosomal shield. b Dorsal, lateral and some part of the ventral cuticle finely wrinkled or covered with scale-like verrucae 
Fig. 2 Amplification of $\mathrm{COI}$ gene produced $378 \mathrm{bp}$ of PCR products. Lane 1: negative control; lane 2: positive control; lanes 3-4: DNA of local Suidasia medanensis (S1a, S2a). Lane M is 100-bp DNA ladder

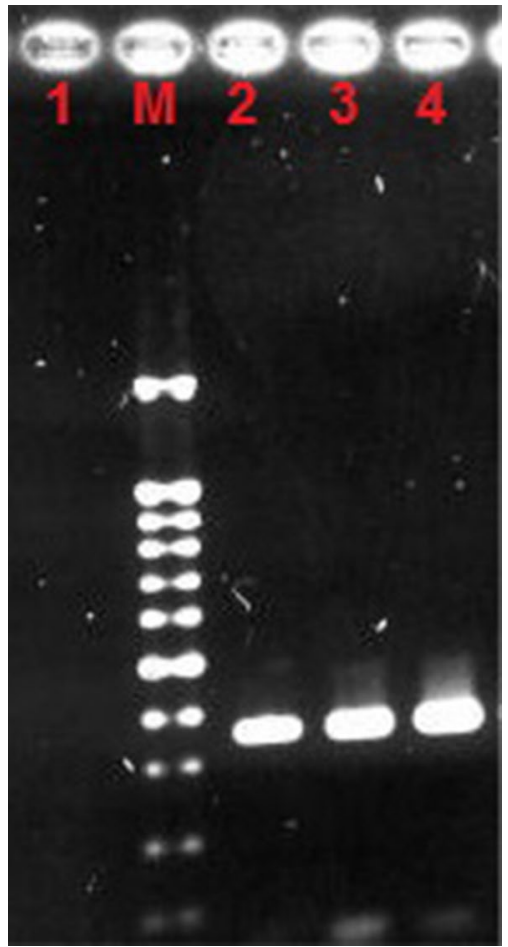

medanensis had 99\% sequence similarity with the S. medanensis (AY525568) available in the GenBank.

In total 327 bp fragments were obtained from the multiple COI gene alignments (Fig. 3). Sequence analysis indicated that $48(14.6 \%)$ variable sites were detected within the COI gene and four $(8.3 \%)$ characters were parsimony informative. Additionally, the conserved sites were constituted by $279(85.3 \%)$ characters showing that the COI segment is a very conserved gene in the mtDNA. In this study, the amino acids asparagine (Asn), histidine (His) and lysine (Lys) were not detected in any of the Suidasia sequences (Fig. 4). Leucine (Leu) was the most abundant $(>43 \%)$ amino acid in all S. medanesis samples.

The phylogenetic tree of $C O I$ indicated that $S$. medanensis from Korea and UK were first apart from the Malaysian mites with a $95 \%$ highly significant bootstrap value (Fig. 5). A monophyletic clade with $85 \%$ bootstrap value was observed for the local species.

\section{Discussion}

The microscopic mite identification has been addressed as a difficult task with the main limitation being the need for expert taxonomic personnel. Moreover, due to their minute size, immature stages often have indistinguishable characters for species identification and they often must be reared to adult stage to be accurately identified. More recently, the availability of DNA sequence data of various mites in international databases has opened the door for simpler molecular-based species identification. In the present study, PCR has successfully produced partial COI sequences of the local $S$. medanensis mites. The 
S1a_COI_F

$\mathrm{S} 2 \mathrm{a} \mathrm{COI}^{-} \mathrm{F}$

AY $5 \overline{2} 556 \overline{8}$ UK

KJ820779 Korea

S1a COI $F$

S2a_COI'

AY $5 \overline{2} 556 \overline{8}$ UK

KJ820779 Korea

S1a_COI_E

S2a COI $\mathrm{F}$

AY $5 \overline{2} 556 \overline{8}$ UR

KJ820779 Korea

S1a_COI_F

S2a COI $\mathrm{F}$

AY $5 \overline{2} 556 \overline{8}$ UK

KJ820779 Korea

S1a_COI $F$

S2a COI $\mathrm{F}$

AY $5 \overline{2} 556 \overline{8}$ UK

KJ820779 Korea

S1a_COI_F

S2a_COI'F

AY $5 \overline{2} 556 \overline{8}$ UR

KJ820779 Korea

S1a_COI_F

$\mathrm{S} 2 \mathrm{a} \mathrm{COI}^{-} \mathrm{F}$

AY $5 \overline{2} 556 \overline{8}$ UK

KJ820779 Korea

S1a_COI $F$

S2a COI $\mathrm{F}$

AY $5 \overline{2} 556 \overline{8}$ UK

KJ820779 Korea

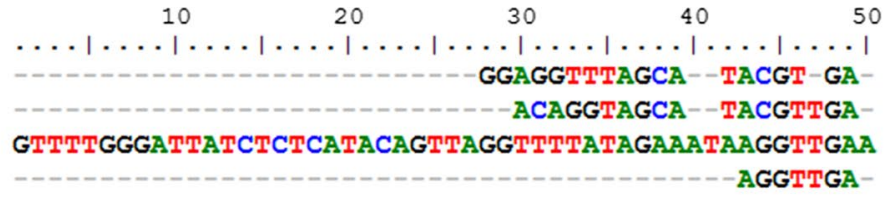

60

70

80

90

100

$\ldots|\ldots| \ldots|\ldots| \ldots|\ldots| \ldots|\ldots| \ldots|\ldots| \ldots|\ldots| \ldots \mid$

CTTTTGGCTCTTT-GGTATAATTTATGCTATAATTTCTATTGGTGTTCT CTTTTGGTTCTTAGGTATAATTTATGCTATAATTCTATTGGTGTTCT ССTTTTGGCTCTTTAGGTATAATTTATGCTATAATTCTATTGGTGTTCT CCTTTTGGCTCTTTAGGTATAATTATGCTATAATTTCTATTGGTGTTCT

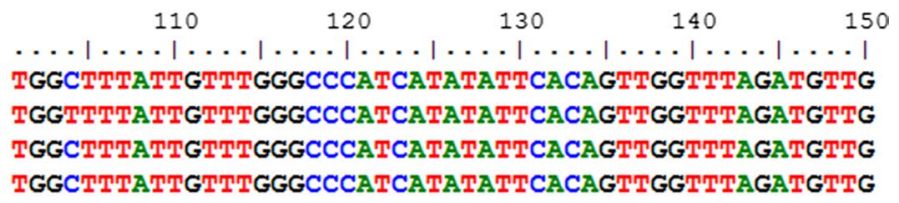

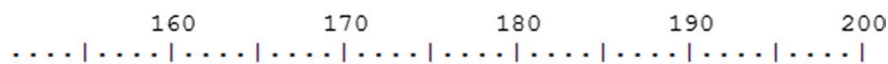
ACACTCGAGCTTATTTTACTGCTGCTACTATAATTATTGCTGTTCCTACG ACACTCGAGCTTATTTTACTGCTGCTACTATGATTATTGCTGTTCCTACG ACACTCGAGCTTACTTTACTGCTGCTACTATAATTATTGCTGTTCCTACG ACACTCGAGCTTACTTTACTGCTGCTACTATAATTATTGCTGTTCCTACG

210

220

230

240

250

$\ldots|\ldots| \ldots|\ldots| \ldots|\ldots| \ldots|\ldots| \ldots|\ldots| \ldots|\ldots| \ldots \mid$ GGGGTTAAGGTTTTTAGTTGATTAGCTACTATGCTTGGAGGTAAGCTTGA GGGGTTAAGGTCTTTAGTTGATTAGCTACTATGCTTGGAGGTAAGCTTGA GGGGTTAAGGTTTTTAGTTGATTGGCTACTATGCTTGGAGGTAAGCTTGA GGGGTTAAGGTTTTTAGTTGATTGGCTACTATGCTTGGAGGTAAGCTTGA

260

270

280

290

$\ldots|\ldots| \ldots|\ldots| \ldots|\ldots| \ldots|\ldots| \ldots|\ldots| \ldots|\ldots|$ TTTTAGTCCTTCTTTTTACTGGTCCTTGGGGTTTGTTTTTCTTTTTACTG TTTTAGTCCTTCTTTTTATTGATCCTTGGGGTTTTTTTTCTTTTTACTG TTTTAGTCCTTCTTTTTACTGGTCCTTGGGGTTGTTTTTCTTTTTACTG TTTTAGTCCTTCTTTTTACTGGTCCTTGGGGTTGTTTTTCTTTTTACTG

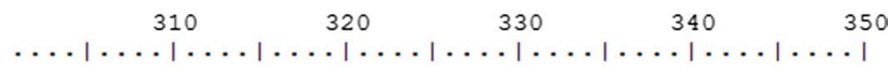
TGGGGGGGTTGACTGGTGTTATTTTGTCTAACTCTTCTCTTGATGTTAGT TGGGGGGGTTGACTGGAGTTATTTTGTCTAACTCTTCTCTTGATGTTAGA TGGGAGGGTTGACTGGTGTTATTTTGTCTAACTCTTCTCTTGATGTTAGT TGGGAGGGTTGACTGGTGTTATTTTGTCTAACTCTTCTCT

$$
\begin{aligned}
& 360 \quad 370 \\
& \ldots|\ldots| \ldots|\ldots| \ldots|\ldots| \ldots \\
& \text { CTTCATGATACTTATTATGTTGTTGCTCC } \\
& \text { CTTCATGATACTTATTATGTTGTTGCTC- } \\
& \text { CTTCATGATACTTATTATGTTGTTGCTC- }
\end{aligned}
$$

Fig. 3 Multiple alignment of local Suidasia medanensis sequence with other sequences from GenBank 


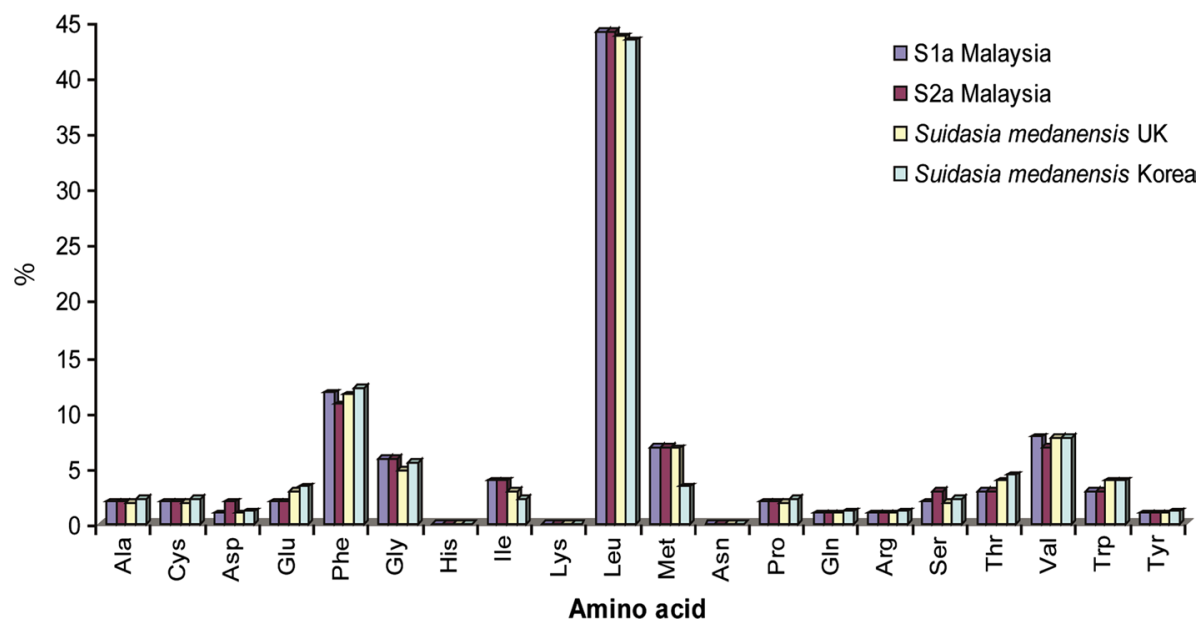

Fig. 4 Amino acid composition (\%) of the COI gene in local Suidasia medanensis compared to those in UK and Korea

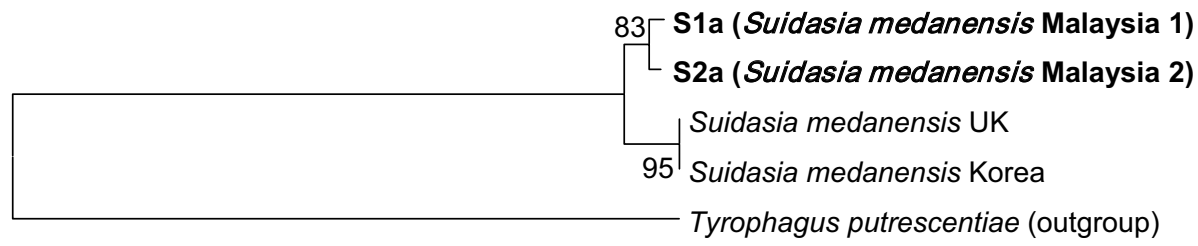

$\stackrel{\longmapsto}{0.01}$

Fig. 5 Phylogenetic tree inferred from COI sequences of Suidasia medanensis by neighbor-joining (NJ) method

COI sequences obtained in this study have been used for species validation of mites as a complement for morphological identification. The results were in concordance to several studies which reported that $C O I$ gene has been often used for molecular identification of dust mites and other astigmatid mites (Navajas et al. 1996; Yang et al. 2011; Khaing et al. 2014).

This study underscores that DNA barcoding using the COI gene can be used to categorize species of dust mites at molecular level. Our results indicated that there was little variation in amino acid percentage of the COI gene between local S. medanensis and those from other parts of the world. This may be due to variation of base composition (deletion or insertion) that occurs mostly through synonymous transitions (Navajas et al. 1996). However, it presumes that the genotypic characterization of the COI gene does not have detectable effects on the phenotypic traits of $S$. medanensis possibly due to the much higher conservation of the length of COI sequences (Khaing et al. 2014).

The NJ analysis of the COI gene positioned the local $S$. medanensis in the phylogenetic tree according to their morphological identification. The families Acaridae (T. putrescentiae) and Suidasiidae (S. medanensis) were distinguished clearly by formation of two major clades. Our study also showed the nearest relatives and provided strong support for 
a single clade of the local mite. Intraspecific genetic distances were relatively smaller (data not shown) for the $C O I$ sequence of both local sequences. Therefore, and because of the limited sample size of the study, intraspecific sequence variation could be investigated further, particularly for those species collected from the field and for species found in other countries worldwide.

In general, the PCR assay can be directly applied in purified mite fractions for a diagnosis or treatment of dust mite allergy, especially when the availability of intact adult mites for morphological identification is limited. Although a single PCR assay may take longer to perform than a morphological identification, the PCR technique has great advantages in terms of turn-around time that can easily identify higher numbers of specimens in a single operation. The present results support that the $C O I$ sequence is a reliable molecular marker for identification and analysis of the phylogenetic relationship of storage mites.

Acknowledgements We would like to thank the Director General of Health Malaysia for his permission to publish this article.

Open Access This article is distributed under the terms of the Creative Commons Attribution 4.0 International License (http://creativecommons.org/licenses/by/4.0/), which permits unrestricted use, distribution, and reproduction in any medium, provided you give appropriate credit to the original author(s) and the source, provide a link to the Creative Commons license, and indicate if changes were made.

\section{References}

Beroiz B, Couso-Ferrer F, Ortego F, Chamorro MJ, Arteaga A et al (2014) Mite species identification in the production of allergenic extracts for clinical use and in environmental samples by ribosomal DNA amplification. Med Vet Entomol 28:287-296

Calderon MA, Linneberg A, Kleine-Tebbe J, de Blay F, Hernandez D, Virchow JC et al (2015) Respiratory allergy due to house dust mites: what do we really know? J Allergy Clin Immunol 136:38-48

Chmielewski W (2009) Pollen pellets as a medium for culture of mites Suidasia pontifica (Oud.) (Acari: Suidasiidae). J Apic Sci 53(1):37-42

Cui Y (2014) When mites attack: domestic mites are not allergens. Parasit Vectors 7:411

Erster O, Roth A, Hadani Y, Shkap V (2013) First detection of Ixodes ricinus on beef cattle in Israel. Vet Parasitol 191(3-4):394-399

Fain A, Philips JR (1978) Notes on the genus Suidasia Oudemans, 1905 with descriptions of a new species from Australia (Acari: Astigmata: Saproglyphidae). Int J Acarol 4(2):115-116

Frati F, Incorvaia C, David M et al (2012) Requirement for acquiring a high-quality house dust mite extract for allergen immunotherapy. Drug Des Dev Ther 6:117-123

Hall TA (2005) Bioedit Version 7.0.4. Department of Microbiology, North Carolina State University

Ho TM (1996) First report of Suidasia pontifica (Acari: Acaridae) in milk. Southeast Asian J Trop Med Public Health 27(4):853-854

Ho TM, Mariana A (1994) The efficacy of a vacuum cleaner for the control of dust mites in mattresses. Trop Biomed 11(2):135-138

Ho TM, Nadchatram M (1984) Distribution of house dust mites in a new settlement in Jengka, Pahang, Malaysia. Trop Biomed 1:49-54

Ho TM, Nadchatram M (1985) Distribution of Dermatophagoides pteronyssinus (Astigmata: Pyroglyphidae) in Cameron Highlands, Malaysia. Trop Biomed 2:54-58

Hughes AM (1976) The mites of stored food and houses. Technical Bulletin 9. Ministry of Agriculture, Fisheries and Food. Her Majesty’s Stationery Office, London, pp. 127-128

Khaing TM, Shim JK, Lee KY (2014) Molecular identification and phylogenetic analysis of economically important acaroid mites (Acari: Astigmata: Acaroidea) in Korea. Entomol Res 44:331-337

Krantz GW, Walter DE, Behan-Pelletier V (2009) A manual of acarology, 3rd edn. Texas Tech University Press, Lubbock

Li HY, Hua T, Yeh WB (2010) Amplification of single bulb mites by nested PCR: species-specific primers to detect Rhizoglyphus robini and R. setosus (Acari: Acaridae). J Asia Pac Entomol 13:267-271 
Li D, Fan QH, Waite DW, Gunawardana D, George S, Kumarasinghe L (2015) Development and validation of Real-Time PCR assay for rapid detection of two-spotted spider mite, Tetranychus urticae (Acari: Tetranychidae). PLoS ONE 10(7):e0131887

Lv J, Wu S, Zhang Y, Chen Y, Feng C, Yuan X, Jia G, Deng J, Wang C, Wang Q, Mei L, Lin X (2014) Assessment of four DNA segments (COI, $16 \mathrm{~S} r \mathrm{DNA}$, ITS2, $12 \mathrm{~S} r D N A)$ for species identification of the Ixodida (Acari: Ixodida). Parasit Vectors 7(1):93-114

Mariana A, Ho TM, Sofian-Azirun M, Wong AL (2000) House dust mite fauna in the Klang Valley, Malaysia. Southeast Asian J Trop Med Public Health 31(4):712-721

Mariana A, Ho TM, Gendeh BS, Iskandar H, Zainuldin-Taib M (2002) First report on sensitization to allergen of a house dust mites, Suidasia pontifica (Acari: Saproglyphidae). Southeast Asian J Trop Med Public Health 31(4):722-723

Mariana A, Heah SK, Wong AL, Ho TM (2010) The occurrence of arthropods in processed rice products in Malaysia. Asian Pac J Trop Med 3(7):552-554

Mariana A, Louis SR, Hamid Z, Ho TM (2011) Scanning electron micrographs of medically important dust mite, Suidasia pontifica (Acari: Astigmata: Saproglyphidae) in Malaysia. Trop Biomed 28(2):275-282

Mercado D, Puerta L, Caraballo L (2001) Life-cycle of Suidasia medanensis (= pontifica) (Acari: Suidasiidae) under laboratory conditions in a tropical environment. Exp Appl Acarol 25:751-755

Navajas M, Fournier D, Lagnel J, Gutierrez J, Boursot P (1996) Mitochondrial COI sequences in mites: evidence for variations in base composition. Insect Mol Biol 5(4):281-285

Puerta L, Lagares A, Mercado D, Fernandez-Caldas E, Caraballo L (2005) Allergenic composition of the mite Suidasia medanensis and cross-reactivity with Blomia tropicalis. Allergy 60:41-47

Sanchez-Borges M, Capriles-Hulett A, Fernandez-Caldas E, Suarez-Chacon R et al (1997) Mite-contaminated foods as a cause of anaphylaxis. J Allergy Clin Immunol 99:738-743

Sanchez-Borges M, Suarez-Chacon R, Capriles-Hulett A, Caballero-Fonseca F, Iraola V, Fernandez-Caldas E (2009) Pancake syndrome (Oral mite anaphylaxis). World Allergy Organ J 2:91-96

Vargas M, Garcia-Varela M, Laclette JP, Perez TM (2005) Application of ITS-2 sequences as marker for identification and phylogenetic inference within the genus Geomylichus (Acari: Listrophoridae). Exp Appl Acarol 35:223-228

Yang B, Cai J, Cheng X (2011) Identification of astigmatid mites using ITS2 and COI regions. Parasitol Res 108:497-503

Yap JMG, Ching MW, Cruz RS, Ramos JDA (2014) Specific IgE against the house dust mite Suidasia pontifica as a risk factor for asthma and allergies in the tropics. Acta Manil 62:1-8 